\title{
A Fast Target Tracking Algorithm Basted on Connected Component Labeling and Grey Value Statistics
}

\author{
Liu Qing, Tang Linbo, Zhao Baojun, Sun Jingle \\ School of Information and Electronics, Beijing Inst. Of Technology
}

\begin{abstract}
A fast target tracking algorithm based on connected component labeling and grey value statistics is proposed. First of all, nonlinear transformation is applied to perform enhancement for targets; and then adaptive threshold is applied to perform pre-processing division for the images; finally, target characteristics which are extracted through connected component labeling and grey value statistics are used to match the gray, the area and position of target, then pinpointing the target. Experimental results on the MATLAB software platform and the SOPC hardware platform show that it can perform real-time track accurately for the infrared small targets.
\end{abstract}

Keywords- Infrared small target, Connected component labeling, Grey value statistics, Real-time performance

\section{INTRODUCTION}

Image processing of high maneuvering targets is a key technology of the guidance system, its main task is providing target seeker with accurate location information. Accuracy and real-time performance are two important indicators of a infrared tracking system. The crossprojection algorithm has the advantage of real time performance and the disadvantage of poor extracting the covered target[1]. Mean shift algorithm has the problem of inaccurate positioning[2]. Multiple target tracking algorithm such as JPDA and MHF has large computation and poor real-time performance $[3,4]$. Connected component labeling algorithm is processing binary image, it has the advantage of the more accurate positioning and the disadvantage of false alarming because of losing the image gray information[5].

This paper expounds a fast target tracking algorithm based on connected component labeling and grey value statistics, First of all, nonlinear transformation is applied to perform enhancement for targets; and then adaptive threshold is applied to perform pre-processing division for the images, connected component labeling algorithm and grey value statistics algorithm are used to track real-time target. The experiment result shows that the fast algorithm expounded by this paper can perfectly solve the problem of the difficult fusion of single target tracking and multi target tracking, poor tracking accuracy, large computation and real-time performance which does not meet the requirement.

\section{ALGORITHN DESIGN}

Flow chart of fast target tracking algorithm is shown in Fig.1(a). Firstly, nonlinear transformation is applied to

perform enhancement for targets; secondly, adaptive threshold is applied to perform pre-processing division for the target, lastly, target characteristics which are extracted through connected component labeling and grey value statistics are used to match and pinpoint the target. The principles of the algorithm are as follows

\section{A. NON-LINEAR TARGET ENHANCEMENT}

For the traditional tracker, a linear quantizing strategy is used to model the probability density function (pdf) of a target model. For our applications, the radiation of infrared targets is often much stronger than those of background and noise. To emphasize the contributions of high gray value components in the image, we propose a nonlinear small infrared target region modeling strategy [6]. For an infrared image with 256 gray levels, a nonlinear quantizing function is defined as

The parameter $M$ is the mean of gray values in the target or the whole infrared image (this variable is set to match the changes of illumination and infrared background) and $l$ is a quantizing sloping factor. $\mathrm{m}$ is the number of bins (i.e. quantizing level). ${ }^{B\left(u_{i}\right)}$ is the quantization value of the pixel intensity at $u_{i}$.

Fig. 2 shows a comparison of the traditional linear quantizing and the proposed nonlinear quantizing. The black square in Fig.2(a) $(64 \times 64$ pixels) is selected to compare quantizing effects. Fig.2(b) and Fig.2(c) are the corresponding gray level histograms quantized by the traditional linear quantizing strategy and our strategy, respectively. It is apparent that our method improves the middle and high gray value components (the quantized values 15 25). Also the distribution of the middle gray value components spreads from the central region to the both sides of the histogram, so the diversity of gray value distribution can be kept after quantizing.

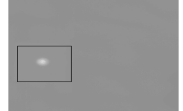

(a)

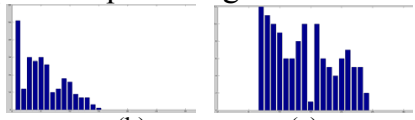

(b)

(c)
Figure 2. Comparisons of the traditional linear quantizing and the proposed nonlinear quantizing (parameters: $M=145$ and $l=2$ )

\section{B. ADAPTIVE IMAGE SEGMENTATION}

As the infrared image under the influence of ambient temperature changes greatly, using fixed or the sole threshold division cannot achieve the good threshold value segmentation results. This paper uses the auto-adapted threshold value segmentation, not only considering the 
overall characteristics of the image, but also taking into account the partial characteristics, to achieve the good threshold value segmentation results.

The auto-adapted threshold infrared small goal segmentation algorithm design is as follows:

1. Compute partial image average gray value $\bar{f}(i, j)$. Uses the entire 1 matrix template and the primitive image $f$ convolution, seeking the image average gray value in a $5 \times 5$ window around estimated point, and $L$ is $5 \times 5$ entire 1 matrix template.

$\bar{f}(i, j)=f \otimes L=\frac{1}{25} \sum_{m=-2}^{2} \sum_{n=-2}^{2} f(i+m, j+n)$

2. Estimate partial variance ${ }(i, j)$. Because calculating the square root is difficult in engineering, using the absolute value average $d(i, j)$ approximate estimate.

$$
d(i, j)=\frac{1}{25} \sum_{m=-2}^{2} \sum_{n=-2}^{2}|f(i+m, j+n)-\bar{f}(i, j)|
$$

3. Supposes $k$ is the threshold coefficient, the Detection threshold computation is as follows:

$$
\begin{gathered}
\text { th }(i, j)= \\
\text { 4. } \\
f(i, j)= \begin{cases}1 & f(i, j)+k \times d(i, j)>t h(i, j) \\
0 & f(i, j)<t h(i, j)\end{cases}
\end{gathered}
$$

\section{ASSOCIATION TARGET TRACKONG}

Grey value statistics algorithm such as mean shift has the advantage of realizing simple and the disadvantage of positioning inaccuracy. Traditional connected component labeling algorithm has the advantage of positioning accuracy and the disadvantage of false alarming because of discarding the image gray information. In this paper, we skillfully combinated connected component labeling and grey value statistics, overcoming the disadvantage of losing the gray information in traditional connected component labeling algorithm and positioning inaccuracy in grey value statistics algorithm.

\section{1) CONNECTED COMPONENT LABELING}

Connected component labeling is a common method of extracting target feature. After the image is processed by connected component labeling, the same label pixels belong to the same target, and then you can calculate the center and area of each target.

\section{2) GREY VALUE STATISTICS}

This paper takes grey value statistics algorithm.All pixels are arranged in ascending order by grey value in the trace window, the first $\quad N 1$ pixels which have the equal quantity with target area $A$ were selected in arranged pixels and extracted grey value statistics, taking the mean value $\mu_{N 1}$ of the first ${ }^{N_{1}}$ pixels as the target mean. Extracting associated ${ }^{N_{2}}$ pixels which have maximum mean value from $N_{1}$ pixels, and calculating the central point coordinate of $N_{2}$ pixels.

\section{Associated basis:}

(1) The grey value difference between adjacent pixels are less than ${ }^{M_{1}}$;

\section{(2) All associated pixels are is in ${ }^{M_{2}}$ neighborhood. 3) TARGET MATCHING}

According to the step 1.3.1 and step 1.3.2, we exact the target average, the target area and central point coordinate and match the gray, the area and position of trace target. The specific matching methods are as follows:

Gray matching: we compare the target average of this frame image with the target average of the previous frame image, judging whether the average difference is in $\delta_{1}$ neighborhood, if the difference is in $\delta_{1}$ neighborhood, grey matching signal is 1 ; else, grey matching signal is 0 .

The method of area matching and position matching is the same as gray matching.

After above 3 steps, there are still more than one suspected targets in the window. According to the most close neighbor principle, the choice is the minimum distance target.

\section{4) JUDGING TARGET AS TRUE OR FALSE.}

According to the step 1.3.3, if grey matching signal,aver matching signal and position matching signal have more than two effective signals, then judging that the target is the real target, carrying on the precise positioning according to the step 1.3.5, else, then judging that the target is the false target, taking the previous frame target location as a target location.

\section{5) PRECISE POSITIONING}

According to the step 1.3.4, if the target is the real target, calculating the difference between the target central point coordinate and target areal coordinates. If the difference is less than the distance between two pixels, then taking the areal coordinates as a target position coordinates, else, taking the average between areal coordinates and central point coordinate as target central location.

\section{3 EXPERIMENTAL RESULT AND ANALYSIS}

The experiment is verified in SOPC hardware platform, its structure diagram is shown in Fig.1(b). Infrared detector transports digital image, enhancing targets, adaptive threshold segmentation, connected component labeling and grey value statistics is carried on in FPGA. Statistical target information is send to CPU by internal double ram, and then targets are matched and pinpointed in Nios II CPU. In the end, the results of handling are sent to the controlling computer.

The fast target tracking algorithm is tested on this signal processing board. Fig. 3 has given an result of an actual image processed by the infrared detector in this system. Multi-target images are simulated in Matlab, three targets move along different elliptical paths. Real movement targets are circled out in the green line, and others are fault targets. Fig. 3 has also given simulation results.

Because all operations are aiming at processing image in tracking Window, and the module are one-way, the simple module repeated use can apply to multiple target tracking and can achieve multi-target tracking.

Proved by system testing, the entire algorithm execution time is smaller than $700 \mu \mathrm{s}$; it is also smaller than $2 \mathrm{~ms}$ 
requested by processing time. This system meets the realtime requirements and the target detection effect is quite ideal.

\section{4 SUMMARIES}

This paper expounds a fast target tracking algorithm based on connected component labeling and grey value statistics, First of all, according to infrared image characteristic, nonlinear transformation is applied to perform enhancement for targets; and then adaptive threshold is applied to perform pre-processing division for the target; target characteristics which are extracted through connected component labeling and grey value statistics are used to match and pinpoint the target, it can perform realtime track accurately for the infrared small targets. The experiment result based on the SOPC hardware platform shows that the algorithm meets the real-time requirements of image processing and possesses good portability. The simulation result based on the MATLAB software platform shows that the algorithm has a multi target tracking effect.

\section{REFRENCES}

[1] WANG Zhao-kui; ZHANG Yu-lin. Algorithm for CCD Star Image Rapid Locating. Chin J Space Sci [J], 2006.26(3), PP:209-214.

[2] Dorin Comaniciu; Senior Member. Kernel-Based Object Tracking. Ieee Transactions On Pattern Analysis And Machine Intelligence, 2003.5, PP:566-577

[3]Lee HK; Ko HS. Predictive estimation method to track occluded multiple objects using joint probabilistic data association filter.ICIAR,2005, PP:852-860.

[4] Chia AYS; Huang WM; Li LY. Multiple objects tracking with multiple hypotheses graph representation.ICPR,2006, PP:638-641.

[5] CHEN Bo-sheng. New connected component labeling algorithm for binary image. Computer Engineering and Application,[J]2007.27(11), PP:2776-2778.

[6] Lei Yang, Wei ping Lu and Jie Yang. A new tracking method for small infrared targets . ICIP,2009, PP:3609-3612.
$B\left(u_{i}\right)=\left\{\begin{array}{l}1, u_{i} \leq \operatorname{Loru}_{i}=0 \\ \operatorname{round}\left(1+\left(1+\sin \left(\frac{l \cdot \pi \cdot\left(u_{i}-M\right)}{256}\right)\right) \cdot \frac{m-1}{2}\right), L<u_{i}<H \\ m, u_{i} \geq H, \text { oru }_{i}=255\end{array}\right.$

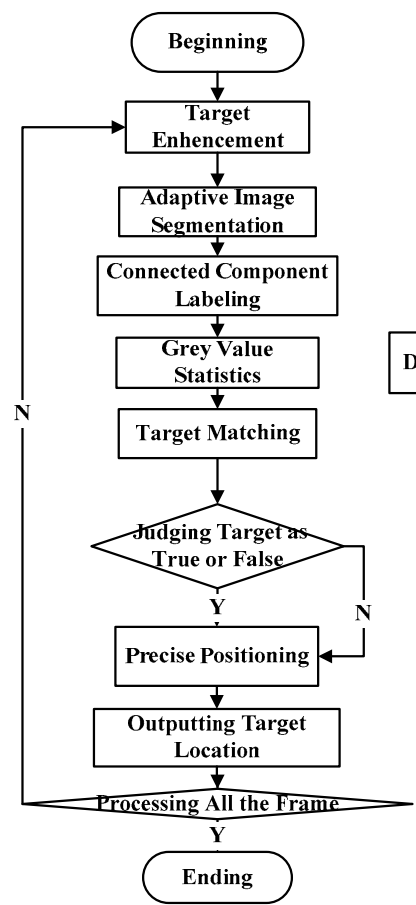

(a)

$$
\begin{aligned}
& H=\text { floor }\left(M+\frac{128}{l}-\frac{256}{m}\right) \\
& L=\operatorname{ceil}\left(M-\frac{128}{l}+\frac{256}{m}\right)
\end{aligned}
$$

figure1. (a) Flow chart of fast target tracking algorithm (b) Structure Block Diagram of SOPC 

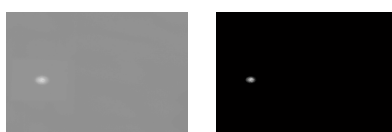

(a) (b)

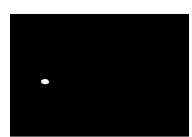

(c)

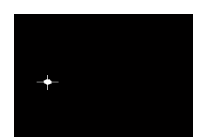

(d)

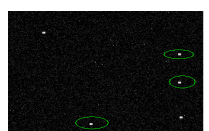

(f)

(g)

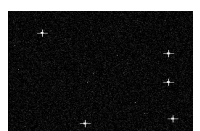

(h)

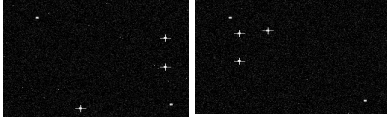

(c)

(d)

Figure3. (a) Original actual image (b) enhanced result (c) Segmentation result (d) Tracking result(e) Multi-objective original image (f) The 1th detection result $(\mathrm{g})$ The 6 th detection result $(\mathrm{h})$ The 157 th detection result 